\title{
The Effect of Hydrogen and Deuterium on the Internal Friction of Deformed Niobium
}

\author{
K. Shibata*† and M. Koiwa*
}

\begin{abstract}
The internal friction of deformed niobium has been measured in a torsion pendulum over a temperature range from $-190^{\circ} \mathrm{C}$ to $+300^{\circ} \mathrm{C}$. By annealing with zirconium foils, the oxygen and hydrogen contents of niobium can be reduced considerably. For such specimens, no $\alpha$-peak has been observed after deformation. Subsequent cathodic charging of hydrogen introduces the $\alpha$-peak. Therefore, the presence of hydrogen is considered to be essential for the occurrence of the $\alpha$-peak.

The addition of deuterium also introduces the $\alpha$-peak at nearly the same temperatures as in the case of hydrogen-charged specimens. In view of a large difference in the diffusivities of hydrogen and deuterium in niobium, the atomsphere dragging may not be the rate-controlling process for the $\alpha$-relaxation.
\end{abstract}

(Received May 1, 1980)

\section{Introduction}

A low temperature relaxation peak termed the $\alpha$-peak ${ }^{(1)}$ is known to appear in bcc refractory metals after plastic deformation. Although a number of experimental investigations have been performed, the origin of the $\alpha$-peak is still a subject of controversy. In a recent paper on niobium, Klam, Schultz and Schaefer ${ }^{(2)}$ have shown that the $\alpha$-peak can be separated into a high temperature component $\alpha_{1}$ and a low temperature component $\alpha_{2}$; the former is assigned to the formation of kink-pairs in non-screw dislocations and the latter to the kink diffusion in screw dislocations.

While these authors consider that hydrogen in metals has only a secondary effect on the $\alpha$-peak, some other authors ${ }^{(3) \sim(5)}$ consider that the presence of hydrogen is essential for the occurrence of the $\alpha$-peak. As to the role of hydrogen, two different models have been suggested. Mazzolai and Nuovo ${ }^{(3)}$ interpreted the $\alpha$-peak as the hydrogen cold-work peak. According to their analysis, various features of the $\alpha$-peak can be successfully explained by the atmosphere dragging model suggested by Schoeck $^{(6)}$. On the other hand, Ferron et al. ${ }^{(5)}$ consider that hydrogen clusters along disloca-

* The Research Institute for Iron, Steel and Other Metals, Tohoku University, Sendai 980, Japan.

$\dagger$ Present address: TDK Electronics Co. Ltd., Tokyo, Japan. tions act as immobile pinning points for dislocations. They tentatively assigned the $\alpha_{1}$ peak to the depinning of non-screw segments, as discussed by Koiwa and Hasiguti( ${ }^{(7)}$ or by Peretz et al. ${ }^{(8)}$, and the $\alpha_{2}$ peak to the depinning of geometrical kinks along the screw components, as discussed by Hasiguti ${ }^{(9)}$.

The aim of this paper is to clarify the role of hydrogen in the $\alpha$-peak in niobium. By annealing niobium with zirconium foils, the oxygen and hydrogen contents can be reduced to a very low level. In such prepared specimens, the $\alpha$-peak is not observed after deformation, while the subsequent cathodic charging of hydrogen introduces the $\alpha$-peak. Therefore, the presence of hydrogen is considered to be essential for the occurrence of the $\alpha$-peak in niobium, as in the case of vanadium reported in previous papers ${ }^{(10)(11)}$. The effect of the addition of deuterium is also examined in comparison to that of hydrogen.

\section{Experimental}

The niobium used in this investigation was obtained from Shin-etsu Kagaku Co., Ltd. in the form of granules. The main metallic impurities are: 400 wt.ppm of Ta, 20 wt.ppm of $\mathrm{Fe}, 10$ wt.ppm of $\mathrm{Al}, \mathrm{Si}, \mathrm{Cr}, \mathrm{Ni}, \mathrm{Ti}$ and $\mathrm{Mo}$, and less than 1 wt.ppm of Mn. An electronbeam-melted ingot was swaged into a bar of $8 \mathrm{~mm}$ in diameter, and then subjected to floating zone refining in an electron beam furnace. 
Wires of $0.6 \mathrm{~mm}$ in diameter were made by swaging and cold drawing from the bar, without intermediate annealings.

Hydrogen charging was performed by cathodic charging at $0^{\circ} \mathrm{C}$. The electrolyte was $0.1 \mathrm{~N} \mathrm{H}_{2} \mathrm{SO}_{4}$ solution and the current density was $100 \mathrm{~A} / \mathrm{m}^{2}$. Deuterium charging was made similarly with $0.1 \mathrm{~N} \mathrm{D}_{2} \mathrm{SO}_{4}-\mathrm{D}_{2} \mathrm{O}$ solution at room temperature.

Specimen wires of about $70 \mathrm{~mm}$ in length were annealed in vacuum-sealed quartz tubes with or without zirconium foils for $2 \mathrm{~h}$ at $1070^{\circ} \mathrm{C}$. The annealing of specimen wires wrapped with zirconium foils (the zirconium treatment) has been found to be effective in removing oxygen as in the case of vana$\operatorname{dium}^{(11)(12)}$, while the bare anneal without zirconium foils increases the oxygen content of specimens; the oxygen content is estimated from the height of the Snoek peak by using the relation determined by Powers and Doyle ${ }^{(13)}$. The residual resistivity ratios of as-drawn, bare-annealed, and zirconiumannealed specimens were 166, 33 and 630 , respectively.

The internal friction was measured in an inverted torsion pendulum ${ }^{(11)(12)}$ over a temperature range from -190 to $+300^{\circ} \mathrm{C}$, usually at a rate of $1.25^{\circ} \mathrm{C} / \mathrm{min}$. Measurements were usually made on heating for temperatures lower than room temperature, and on cooling for higher temperatures. The measuring frequency is typically about $1.5 \mathrm{~Hz}$, and the strain amplitude in the maximum surface shear strain is $7 \times 10^{-6}$. The zero point drift of the torsion pendulum was optically detected and recorded on a chart paper during measurements of internal frictions.

\section{Results and Discussion}

\section{The internal friction of as-drawn and annealed specimens}

Figure 1 shows the internal friction of a specimen drawn to $0.6 \mathrm{~mm}$ in diameter from a zone-refined bar without any intermediate annealings. A large $\alpha$-peak appears at about $-150^{\circ} \mathrm{C}$. A cooling run curve after heating up to $350^{\circ} \mathrm{C}$ is also shown in the figure. From the

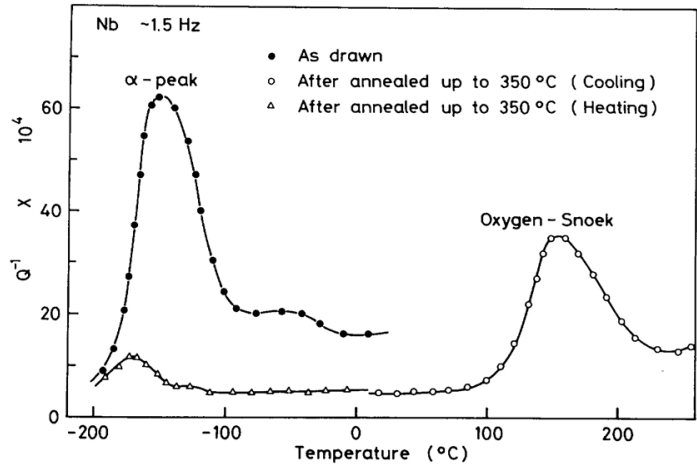

Fig. 1 The internal friction of an as-drawn specimen.

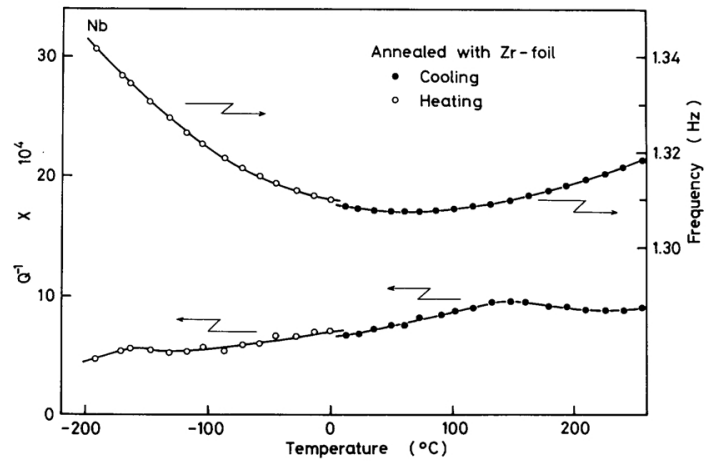

Fig. 2 The internal friction and the vibrational frequency of a specimen prepared by the zirconium treatment.

height of the Snoek peak at $154^{\circ} \mathrm{C}$, the oxygen content is estimated to be about 600 at.ppm. After this annealing, the $\alpha$-peak is greatly reduced, but the residue is still observed at $-170^{\circ} \mathrm{C}$.

Figure 2 shows the internal friction and the vibrational frequency of a zirconium-annealed specimen. The data shown here were taken after heating up to $350^{\circ} \mathrm{C}$ in situ in order to eliminate a possible effect of handling. The oxygen content is estimated to be about 20 at.ppm. The elastic modulus of niobium has been known to exhibit an anomalous temperature dependence ${ }^{(14)}$.

\section{The effect of deformation and hydrogen addition}

After the measurement shown in Fig. 2, the specimen has been subjected to torsional deformation of $19 \%$ in the surface shear strain at room temperature. As shown in Fig. 3, 


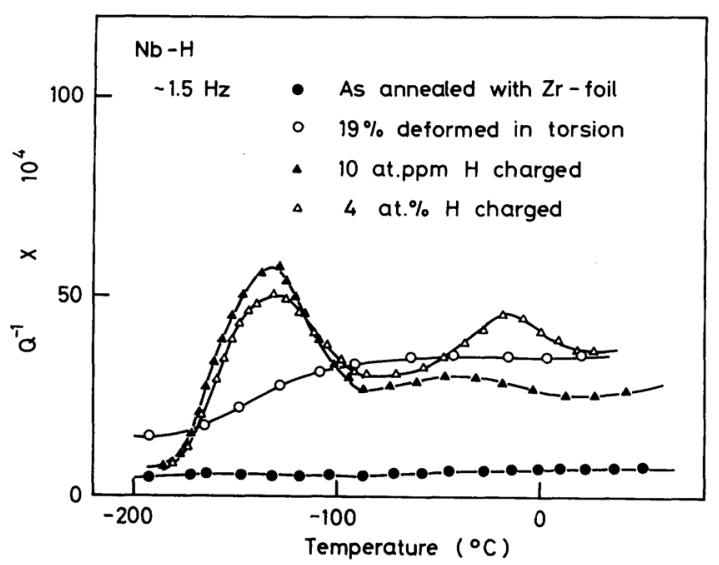

Fig. 3 The effects of deformation and the addition of hydrogen on the internal friction. The specimen is the same as that for Fig. 2.

the $\alpha$-peak is not observed. Subsequent cathodic charging of hydrogen introduces the $\alpha$-peak. The amount of charged hydrogen is estimated from the increase in the electrical resistivity by using the value $0.64 \mu \Omega \cdot \mathrm{cm} / \mathrm{at} . \% \mathrm{H}^{(15)}$. A further addition of hydrogen to about 4 at. $\%$ does not change or slightly decrease the height of the $\alpha$-peak, and introduces the precipitation peak at about $-15^{\circ} \mathrm{C}$. The frequency and the self-twisting of the specimen simultaneously measured with the internal friction are shown in Fig. 4. In contrast to the case of vanadium, the dissolution or precipitation temperature cannot be clearly defined from the observation of self-twisting as a function of temperature; for vanadium a sharp knick was observed (see, e.g. Fig. 4 of Ref. (11)).

\section{Hydrogen pick-up}

Va group metals are known to absorb hydrogen easily. The degassing of these metals in ultra high vacuum does not necessarily assure the absence of hydrogen in the specimen at the time of measurements of internal friction. The zirconium treatment adopted in the present experiment is expected, from thermodynamic consideration $^{(16)}$, to yield niobium specimens with an extremely low hydrogen content. However, the treatment is not always successful as judged from the occasional appearance of the trace of the $\alpha$-peak after plastic deformation. The reabsorption of hydrogen might occur

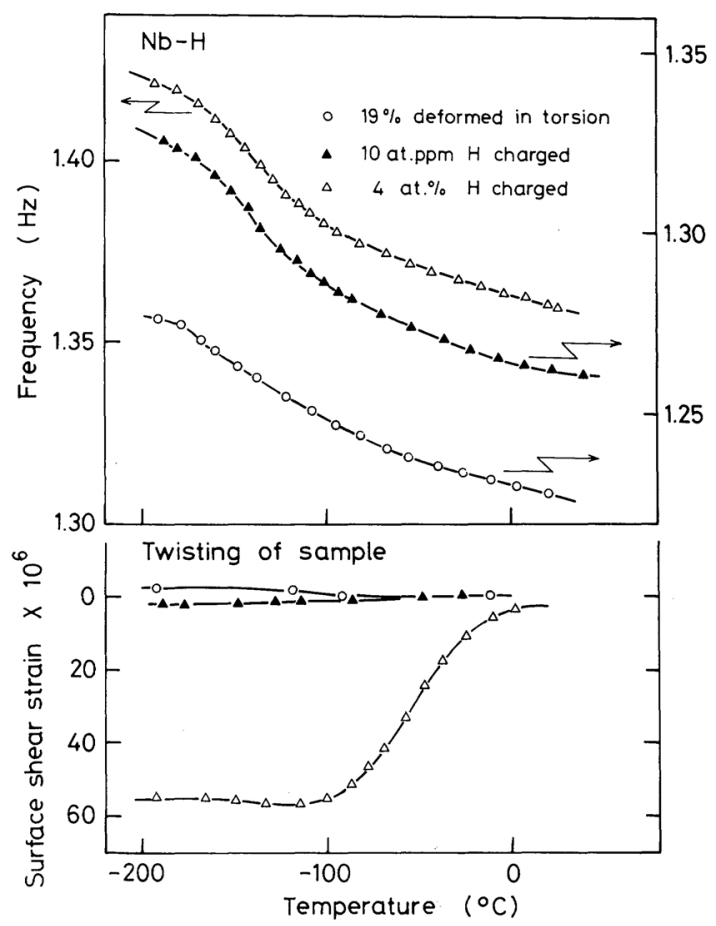

Fig. 4 The vibrational frequency and the self-twisting simultaneously measured with the internal friction measurements shown in Fig. 3.

when the specimen is exposed to air after the treatment, or during handling.

Various mechanical treatments are known to introduce hydrogen. Abe, Yoshinaga and Morozumi $^{(17)}$ have shown that slicing by a wheel-cutting machine with a cooling-water jet introduces a considerable amount of hydrogen into $\mathrm{V}, \mathrm{Nb}$ and Ta. Lang and Bressers $^{(18)}$ have observed that vanadium picks up hydrogen to such amounts as can be detected by differential scanning calorimetry, after dry or wet grindings.

A similar experiment is also performed as shown in Fig. 5. First, the internal friction is measured for a deformed specimen prepared by the zirconium treatment; the $\alpha$-peak is not observed. The surface of the specimen is then filed with Emery paper in the water to remove zirconium foils adhered to the surface. After this treatment, the $\alpha$-peak is clearly observed, indicating the absorption of hydrogen. 


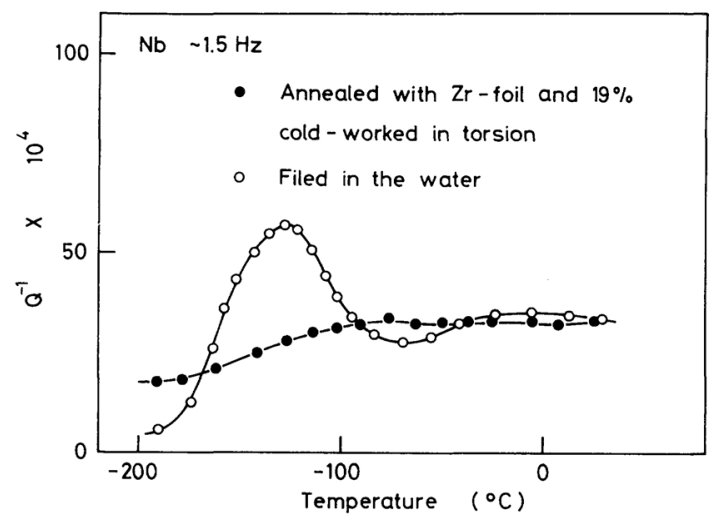

Fig. 5 The effect of filing in the water on the internal friction spectrum.

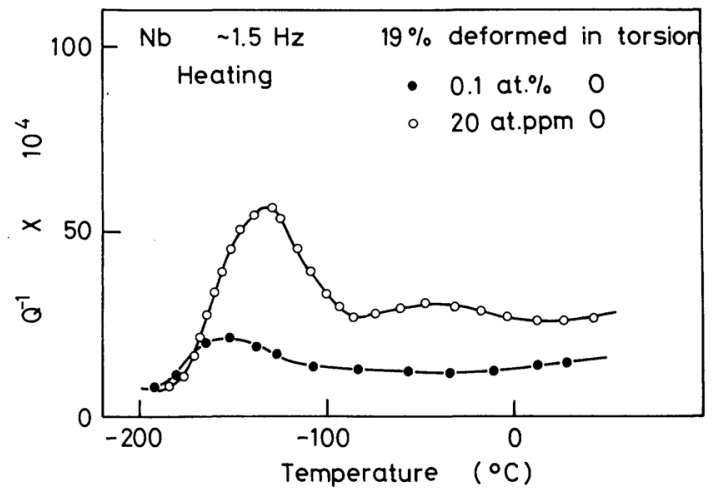

Fig. 6 The effect of oxygen on the height of the $\alpha$-peak.

\section{Effect of oxygen on the height of the $\alpha$-peak}

Figure 6 compares the height of the $\alpha$-peak in two specimens with different oxygen contents. The specimen with about 0.1 at. $\%$ oxygen had been annealed without zirconium foils. After deformation at room temperature, a small $\alpha$-peak appears at about $-160^{\circ} \mathrm{C}$, which is considered to be due to the presence of hydrogen. The hydrogen content after the bare anneal is not known, but is considered not to be very much different from that before the anneal; the hydrogen content may be of the same order as that of the specimen in Fig. 1. For the sake of comparison, the data in Fig. 3 (zirconiumannealed, deformed and then hydrogen-charged to 10 at.ppm) are shown together. As in the case of vanadium, oxygen seems to suppress the $\alpha$-peak. The temperature of the peak is

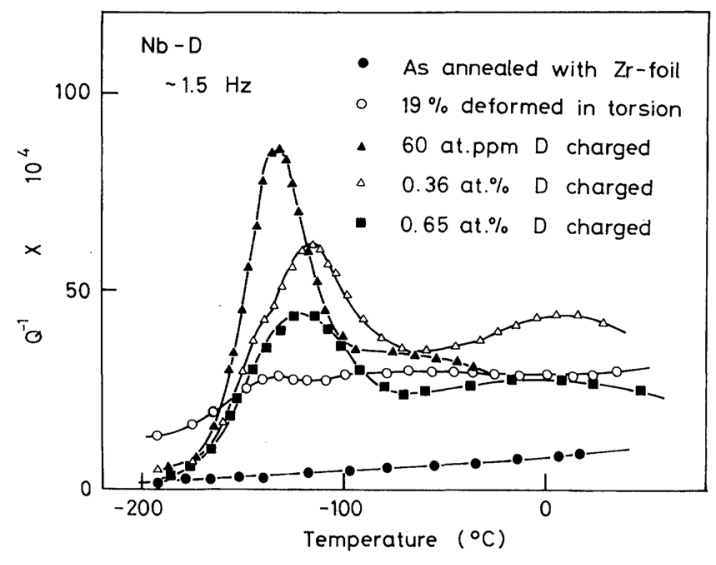

Fig. 7 The effect of deuterium on the $\alpha$-peak.

lower in the specimen of a high oxygen content, being consistent with the observation by Amateau et al. ${ }^{(19)}$

\section{Effect of deuterium addition}

A zirconium-annealed specimen was subjected to successive charging of deuterium, after deformed $19 \%$ at room temperature. As shown in Fig. 7, a trace of the $\alpha$-peak seems to exist before deuterium charging, which may be due to residual hydrogen. With the addition of 60 at.ppm deuterium, a large $\alpha$-peak appears, but further additions rather suppress the peak. Internal friction spectra of niobium containing deuterium (or hydrogen) are generally affected by the precipitation of the deuterides, and the curves in Fig. 7 are considered to have some contribution of the precipitation peak. The behaviour of the precipitation peak is now under investigation and will be the subject of a separate paper.

The effects of addition of a similar amount of hydrogen or dueterium are compared in Fig. 8. In this experiment, two specimens subjected to a zirconium-anneal are used; before charging, the internal friction spectra are virtually the same for the two specimens. Although the height and the temperature of peaks are somewhat different, general features are very similar for the $\mathrm{H}$ - and the $\mathrm{D}$-charged specimens.

\section{Origin of the $\alpha$-peak}

The result of the present experiment clearly 


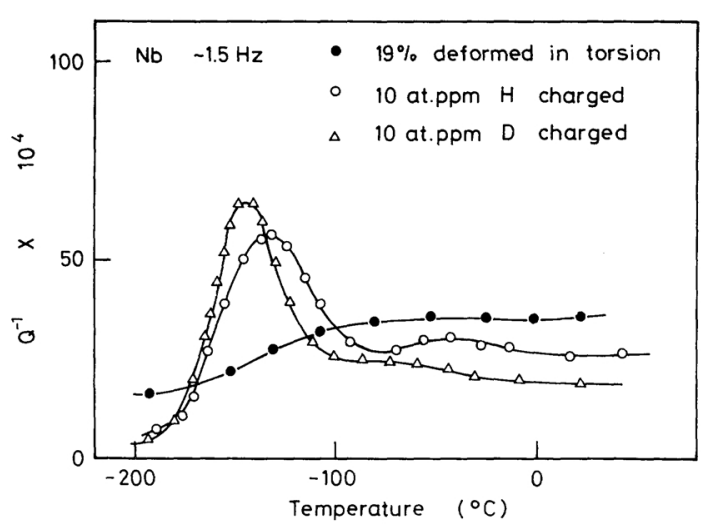

Fig. 8 Comparison of the $\alpha$-peak in hydrogen- and deuterium-charged specimens. The curve for the hydrogen-charged specimen is the same as that in Fig. 3.

supports the view that the presence of hydrogen (or deuterium) is essential for the occurrence of the $\alpha$-peak in niobium ${ }^{(3) \sim(5)}$. Mazzolai and Nuovo ${ }^{(3)}$ explained the $\alpha$-peak by the atmosphere dragging model suggested by Schoeck ${ }^{(6)}$.

Here, we shall examine what kinds of difference should be expected, with the Schoeck model, for the $H \alpha$-peak and the $D \alpha$-peak. According to the model, the relaxation time $\tau$ is proportional to $C_{d} / D$, where $C_{d}$ is the hydrogen atomic fraction along the dislocation line, and $D$ is the hydrogen diffusion coefficient.

The values of the diffusion coefficients for hydrogen and deuterium in $\mathrm{Nb}$ are known to be considerably different, and the followings are suggested as the best values ${ }^{(20)}$.

$$
\begin{gathered}
\text { Hydrogen: } D_{0}=0.9 \times 10^{-4} \mathrm{~cm}^{2} \mathrm{~s}^{-1}, \\
U=0.068 \mathrm{eV}\left(T<-50^{\circ} \mathrm{C}\right) \\
\text { Deuterium: } D_{0}=5.2 \times 10^{-4} \mathrm{~cm}^{2} \mathrm{~s}^{-1}, \\
U=0.127 \mathrm{eV}
\end{gathered}
$$

The hydrogen concentration $C_{d}$ along dislocation lines is dependent on the hydrogendislocation binding energy $E_{B}$. Since the changes in the lattice dimension of niobium with the addition of hydrogen and deuterium are substantially the same ${ }^{(21)}$, the values of $E_{B}$ may be also similar. The activation energy for the $\alpha$-peak is given by the sum of $U$ and $E_{B}$. The expected peak temperatures of the $\mathrm{D} \alpha$-peak have been calculated for values of $E_{B}$ ranging from 0.05 to $0.2 \mathrm{eV}$ by taking the peak tem- perature of the $\mathrm{H} \alpha$-peak as $-130^{\circ} \mathrm{C}$ :

$$
\begin{array}{lrrrr}
E_{B}(\mathrm{eV}) & 0.05 & 0.10 & 0.15 & 0.20 \\
T_{m}\left({ }^{\circ} \mathrm{C}\right) & -91 & -102 & -108 & -112
\end{array}
$$

While the above calculation predicts that the D $\alpha$-peak should appear at temperatures $18 \sim$ $39^{\circ} \mathrm{C}$ higher than the $\mathbf{H} \alpha$-peak, no such difference has been observed experimentally. The result in Fig. 8 shows even an opposite trend, although this should not be taken too seriously; the peak temperature is very sensitive to various factors. Therefore, it is likely that the atmosphere dragging is not the rate controlling process for the $\alpha$-relaxation. The above analysis seems to be in favour of the idea by Ferron et al. ${ }^{(5)}$ that hydrogen atoms or clusters act as immobile pinning points for dislocations.

The effect of hydrogen addition on the low temperature hardening has been studied by Ravi and Gibala ${ }^{(22)}$ and by Chen and Arsenault $^{(23)}$. The former authors ascribe the hardening to hydrogen trapped by other point defects, while the latter authors consider that the major contribution to the strengthening is the stress-induced reorientation of hydrides. However, it is still possible that hydrogen atoms act as immobile pinning points for dislocations at such low stress levels as used for internal friction measurements. Further investigations are required to clarify the role of hydrogen in the $\alpha$-relaxation process.

\section{Summary and Conclusions}

(1) The internal friction of deformed niobium has been measured in a torsion pendulum between $-190^{\circ} \mathrm{C}$ and $+300^{\circ} \mathrm{C}$.

(2) Both the oxygen and hydrogen contents in niobium can be reduced by annealing with zirconium foils.

(3) The $\alpha$-peak in niobium requires the presence of hydrogen (or deuterium).

(4) The addition of oxygen suppresses the height of the $\alpha$-peak, and lowers the peak temperature.

(5) The peak temperatures of the $\mathrm{H} \alpha$-peak and the $\mathrm{D} \alpha$-peak are not very different. This observation is difficult to explain by the atmosphere dragging model by Schoeck; 
hydrogen or deuterium seems to act as immobile pinning points for dislocations.

\section{Acknowledgements}

The authors wish to express their thanks to Professor M. Hirabayashi for continued interests and encouragements, and to Professor P. G. Bordoni for reading the manuscript and valuable comments. Their thanks are also due to Drs. O. Yoshinari and S. Tanaka for advice and discussions. They also wish to thank Messrs. S. Ono, K. Wako and K. Obara for the assistance in the specimen preparation. This work is partly supported by the Grant in Aid for Scientific Research from the Ministry of Education.

\section{REFERENCES}

(1) R. H. Chambers: Physical Acoustics, ed. by W. P. Mason, Academic Press, New York (1966), Vol. III A, p. 123.

(2) R. Klam, H. Schultz and H.-E. Schaefer: Acta Met., 27 (1979), 205.

(3) F. M. Mazzolai and M. Nuovo: Solid st. Communs., 7 (1969), 103.

(4) G. Ferron, M. Quintard and J. de Fouguet: Sixth Int. Conf. on Int. Friction and Ultrasonic Attenuation in Solids, Tokyo, (1977).

(5) G. Ferron, M. Quintard and P. Mazot: Scripta Met., 12 (1978), 623.

(6) G. Schoeck, Acta Met., 11 (1963), 617.
(7) M. Koiwa and R. R. Hasiguti: Acta Met., 13 (1965), 1219.

(8) J. Perez, P. Peguin and P. Gobin: J. de Phys., 32, suppl. 7 (1971), 127.

(9) R. R. Hasiguti: J. Phys. Soc. Japan, 18, suppl. 1 (1963), 114.

(10) K. Shibata, M. Koiwa and O. Yoshinari: Trans. Jap. Inst. Metals, 19 (1978), 491.

(11) M. Koiwa, K. Shibata and O. Yoshinari: Trans. Jap. Inst. Metals, 21 (1980), 191.

(12) O. Yoshinari, M. Koiwa, H. Asano and M. Hirabayashi: Trans. Jap. Inst. Metals, 19 (1978), 171.

(13) R. W. Powers and M. V. Doyle: J. Appl. Phys., 30 (1959), 514.

(14) H. Albert and I. Pfeiffer: Z. Metallk., 58 (1967), 311.

(15) G. Pfeiffer and H. Wipf: J. Phys., F6 (1976), 167.

(16) M. Koiwa and K. Shibata: J. Less Common Metals, 72 (1980), 125.

(17) K. Abe, H. Yoshinaga and S. Morozumi: Trans. Jap. Inst. Met., 17 (1976), 551.

(18) E. Lang and J. Bressers: Z. Metallk., 67 (1976), 66.

(19) M. F. Amateau, T. E. Mitchell and R. Gibala: Phys. Status Solidi, 36 (1969), 407.

(20) J. Völkl and G. Alefeld: Hydrogen in Metals, ed. by G. Alefeld and J. Völkl, Springer-Verlag, Berlin (1978), Vol. 1, p. 321.

(21) H. Peisl: ibid., (1978), Vol. 1, p. 54.

(22) K. V. Ravi and R. Gibala: Met. Trans., 2 (1971), 1219.

(23) C. C. Chen and R. J. Arsenault: Acta Met., 23 (1975), 255. 